\title{
Cerebrospinal meningitis: a global disease with regional variability?
}

\author{
Jason L. Siegel \\ Department of Neurology, Department of Critical Care Medicine, Department of Neurosurgery, Mayo Clinic, Jacksonville, Florida, USA
}

\begin{abstract}
Introduction. In this edition, Szymanski et al. present the results of their retrospective study of the clinical differences between patients with meningococcal meningitis and pneumococcal cerebrospinal meningitis at the Regional Specialistic Hospital in Wroclaw, Poland.

Clinical reflections. The authors found that compared to patients with N. meningitidis, patients with S. pneumoniae were older, more frequently had chronic comorbidities, and had higher rates of pneumonia, longer hospitalisations, and higher mortality. Patients with N. meningitidis had higher rates of haemorrhagic rash and DIC.

Clinical implications. These characteristics and outcomes reflect previous reports from Western Europe and the United States.

Key words: Meningitis, health care outcomes, sepsis, risk factors

(Neurol Neurochir Pol 2020; 54 (1): 6-7)
\end{abstract}

Bacterial meningitis has an incidence in developed countries of $0.7-0.9$ per 100,000 , but remains as high as 40 per 100,000 in developing countries [1]. Previously described risk factors include an immonocompromised state such as age (infants and elderly), hyposplenia, HIV/AIDS, cancer, diabetes mellitus, alcoholism, and organ transplantation [2]. Before the advent of antibiotics, meningitis was typically fatal. But with the advent of pathogen-specific antimicrobials, vaccinations towards encapsalated bacteria, and corticosteroids, survival is now nearly $80 \%$ [3-5]. Although Europe, especially the Netherlands, has led the way in clinical meningitis research, the differing health systems across the continent may change the clinical characteristics and outcomes of meningitis.

Szymanski et al. conducted a retrospective study over a 20-year period at the J. Gromkowski Regional Specialistic Hospital in Wroclaw, Poland [6]. Their study reflected several previously reported characteristics regarding patients with $S$. pneumoniae and N. meningitidis. Pneumococcal meningitis patients were more likely to be older and to have chronic disease, to experience a longer delay in diagnosis, and to have lower levels of white blood cells in their CSF, higher rates of pneumonia, longer hospitalisations, and higher mortality. Meningococcal meningitis patients were far more likely to present with headache and vomiting, with over $60 \%$ having a haemorraghic rash, and around 30\% developing DIC. No patients with menigococcal meningitis died during their study, whereas about $21 \%$ of the pneumococcal patients died. These are numbers that reflect Western European and USA experience.

The clinical course of meningitis, including mortality, can vary geographically due to variations in bacterial serotypes, rates of vaccination, rates of immunocompromised states, access to acute healthcare and hospitals, and genetic susceptibility. Most epidemiological work has been done in either Western Europe or North America or in developing countries of South America, Africa, and Asia. This is the first study to have investigated the differences between these diseases in the Polish population. This study shows that Polish patients at this hospital present with typical clinical findings, receive quick and appropriate treatment including corticosteroids (95\%), and have similar mortality outcomes to those found in previous studies.

\section{References}

1. Brouwer M, Beek Dv. Epidemiology of community-acquired bacterial meningitis. Current Opinion in Infectious Diseases. 2018; 31(1): 78-84, doi: 10.1097/qco.0000000000000417. 
2. Adriani KS, Brouwer MC, van de. Risk factors for community-acquired bacterial meningitis in adults. Neth J Med. 2015; 73(2): 53-60.

3. Buchholz G, Koedel U, Pfister HW, et al. Dramatic reduction of mortality in pneumococcal meningitis. Crit Care. 2016; 20(1): 312, doi: 10.1186/s13054-016-1498-8, indexed in Pubmed: 27716447.

4. Brouwer MC, McIntyre P, Prasad K, et al. Corticosteroids for acute bacterial meningitis. Cochrane Database Syst Rev. 2010(9): CD004405, doi: 10.1002/14651858.CD004405.pub3, indexed in Pubmed: 20824838.
5. Brouwer MC, Heckenberg SGB, de Gans J, et al. Nationwide implementation of adjunctive dexamethasone therapy for pneumococcal meningitis. Neurology. 2010; 75(17): 1533-1539, doi: 10.1212/ WNL.0b013e3181f96297, indexed in Pubmed: 20881273.

6. Szymański W, Simon KA, Rorat M. Differences in the courses of meningococcal and pneumococcal cerebrospinal meningitis. Neurol Neurochir Pol. 2020; 54(1): 39-46, doi: 10.5603/PJNNS.a2020.0002, indexed in Pubmed: 31956973. 